Original Research Article

\title{
A comparative study of mebeverine and synbiotic combination in patients with diarrhoea predominant Irritable Bowel Syndrome in a Medical College in South India
}

\author{
G. Sujatha ${ }^{1}$, M. Dhanasekaran ${ }^{1}$, Syed Shuja Qadri*², S. Jeevithan²
}

\begin{abstract}
${ }^{1}$ Department of Pharmacology, Government Mohan

Kumaramangalam Medical College and Hospital, Salem, Tamil Nadu, India

${ }^{2}$ Department of Community Medicine, Karpagam Faculty of Medical Sciences and Research, Coimbatore, Tamil Nadu, India
\end{abstract}

Received: 11 November 2016 Accepted: 01 December 2016

\section{*Correspondence to: \\ Dr. Syed Shuja Qadri, \\ Email: ssaqadri@yahoo.com}

Copyright: (C) the author(s), publisher and licensee Medip Academy. This is an openaccess article distributed under the terms of the Creative Commons Attribution NonCommercial License, which permits unrestricted noncommercial use, distribution, and reproduction in any medium, provided the original work is properly cited.

\begin{abstract}
Background: Irritable bowel syndrome (IBS) is a chronic, episodic functional gastrointestinal disorder characterized by abdominal pain / discomfort and altered bowel habits. Though it is considered as a functional disorder, the burden of the disease to the patients is very high and the quality of life becomes miserable. Currently available IBS therapies are mainly symptom oriented and have limited efficacy. Various studies had done so far which provide a clear rationale for the use of Synbiotic in this disorder. The objective of the study includes, this study was planned to compare the efficacy of Mebeverine + Synbiotic combination with Mebeverine and Synbiotic monotherapy in patients with diarrhoea predominant irritable bowel syndrome.

Methods: The study was done in Department of Medical Gastroenterology, Rajiv Gandhi Government Hospital, Chennai for duration of one year. Patients with Irritable Bowel Syndrome (diarrhea predominant type), diagnosed within 1 year and attending outpatient department were taken. A randomized, Phase III, prospective, interventional, open label, outpatient, comparative study design was done. A total of 60 patients divided into 3 groups were finally selected for the study purpose.

Results: Twelve weeks after completion of active drug therapy, the Mebeverine + Synbiotic combination improved all the symptoms of IBS except abdominal pain. Further it was evident that combination therapy had significant remission in stool frequency and consistency when compared with other groups.

Conclusions: Combination of Mebeverine + Synbiotic is more effective in improving most of the troublesome symptoms in patients with diarrhea predominant irritable bowel syndrome than other therapies and also in maintaining remission, in terms of frequency and consistency of stools.
\end{abstract}

Keywords: Irritable bowel syndrome, Probiotic, Synbiotic

\section{INTRODUCTION}

Irritable bowel syndrome (IBS) is a chronic, episodic functional gastrointestinal disorder characterized by abdominal pain / discomfort and altered bowel habits such as constipation, diarrhea or alternating periods of both. IBS symptoms last for many years, with a mean duration of 10 years. ${ }^{1}$ Though it is considered as a functional disorder, the burden of the disease to the patients is very high and the quality of life in patients with IBS is miserable. Currently available IBS therapies such as antidiarrheal, antispasmodic and 5HT receptor agonist /antagonist are mainly symptom oriented and often are of limited efficacy regarding the overall complaints. Studies have shown that an imbalance in the intestinal flora may be associated with IBS. ${ }^{2}$ Disordered microflora may contribute to GI symptoms through altered colonic fermentation resulting in the increased formation of gas and an abnormal pattern of short chain fatty acids.

Synbiotic denotes a synergistic combination of probiotic and prebiotic, the viable beneficial bacteria and their selective substrates. With this combination, the beneficial 
bacteria that survive their transit through the stomach, can grow quickly and competitively because of the presence of the selective substrate and establish their predominance. Combining probiotic with prebiotic would improve the survival of bacteria crossing stomach, thus enhancing their effects in the large bowel. Accordingly this provides a clear rationale for the use of Synbiotic in this disorder.

In normal individuals high amplitude propagated contractions (HAPCs) produce propulsive contractions in the colon and produce mass movements of feces and defecation. In IBS, diarrhea can occur from multiple colonic mechanisms including increased HAPCs, increased gastrocolic motility or rectal hypersensitivity. ${ }^{5}$ Abdominal pain in IBS may be associated with HAPCs. ${ }^{6}$ In healthy persons, the Migrating Motor Complex (MMC) repeats every 2 hours in small Bowel. The main purpose of $\mathrm{MMC}$ is to facilitate the transportation of undigested substances from small bowel. In diarrhea predominant IBS patients the migrating motor complex cycle length is reduced. ${ }^{7}$

Mebeverine is a musculotropic antispasmodic agent which directly acts on the gut smooth muscle cell. ${ }^{8}$ It exerts antispasmodic action by reducing sodium ion permeability of smooth muscles. Secondly, it reduces potassium ion efflux thus avoiding hypotonia. Since mechanism of action of mebeverine is not exerted by autonomic nervous system, anticholinergic side effects are rare. ${ }^{9}$

Various studies done so far have been done with Synbiotic alone or Symbiotic with Probiotic combination or Mebeverine alone. So present study was planned to compare and evaluate the efficacy of Mebeverine +Synbiotic combination therapy in patients with diarrhea predominant IBS with Mebeverine monotherapy and Synbiotic monotherapy.

\section{METHODS}

The study was carried out in the in Department of Medical Gastroenterology, Rajiv Gandhi Government Hospital, Chennai for duration of one year. All the patients with Irritable Bowel Syndrome (18-60 yrs), diagnosed within 1 year and attending outpatient department were taken for the study purpose.

\section{Study design}

A randomized, Phase III, prospective, interventional, open label, outpatient, comparative study design was adopted.

Sample size was about totally 60 patients (20 patients in three groups).

\section{Inclusion criteria}

1. Patients diagnosed to have Irritable Bowel Syndrome (diarrhea predominant type) within 1 year.

2. Both genders.

3. 18 to 60 years.

\section{Exclusion criteria}

1. Pregnant and lactating women.

2. Peritonitis with sepsis.

3. Patients with infectious, ischemic, radiation induced and chemical colitis.

4. Complications of previous abdominal surgery.

5. History of antibiotic administration in the past one month.

6. Malignancy, thromboembolic disease.

7. Patients with hypersensitivity for Probiotics, Synbiotic and Mebeverine.

8. Patients with chronic or severe respiratory, cardiovascular, CNS, endocrine and other gastrointestinal disorders.

\section{Strategy}

Patients diagnosed with IBS were explained in detail about the study procedure, purpose and its benefits. They were assured of utmost confidentiality. Written informed consent was obtained from the patients willing to participate in the study, in the prescribed format in the regional language. If the patient was illiterate, left thumb impression was obtained in the presence of an impartial witness.

\section{Screening and recruitment}

Patients who had given the informed consent for participation in the study were screened by medical history, physical examination, clinical examination and laboratory investigations. Finally 60 patients who fulfilled the inclusion criteria were recruited for the study purpose.

\section{Randomization}

Patients were randomized to either of the study groups and received respective medications:

- Group A: Tab. Mebeverine $135 \mathrm{mg}$ twice daily for 12 weeks.

- Group B: Cap. Synbiotic twice daily for 12 weeks.

- Group C: Tab. Mebeverine $135 \mathrm{mg}$ twice daily for 12 weeks along with Cap. Synbiotic twice daily for 12 weeks.

Patients were instructed to take Tab. Mebeverine 20 minutes before food and cap. Synbiotic after food. The patients were advised to take their medicines regularly. The necessity for compliance to the regimen was 
explained. Any adverse events experienced by the patients were to be reported at the next visit.

Assessment and Follow up: The patients were requested to come for review every 4th week during treatment period and also during follow up period for 24 weeks. The following parameters were assessed during each visit:

- Abdominal pain

- Frequency of stools

- Consistency of stool

- Abdominal bloating

The following laboratory investigations were done at the baseline and at the end of 12 weeks:

- Biochemistry

- Blood urea

- Serum creatinine

- SGOT

- $\quad$ SGPT

- Hematology

- Hemoglobin

- Total count

The patients were followed for a period of 12 weeks after completion of active drug therapy.

\section{Ethical consideration}

The study was conducted after obtaining permission from the Institutional Ethics Committee and it was done in accordance with the declaration of Helsinki and Good Clinical Practice (GCP) guidelines. Informed and written consent from all the participants was taken. Confidentiality was maintained at all times during the course of the study. There was no financial burden to the participants.

\section{Data analysis}

The data thus obtained was analyzed using SPSS software (version 20). The statistical tools were described in terms of percentage among various categories of patients. Illustration was done using pie charts and bar graphs. Appropriate statistical methods (Chi-square test) were applied as per requirement. $\mathrm{P}$ value $\leq 0.05$ was considered significant.

\section{RESULTS}

A hospital based study was conducted to compare and evaluate the efficacy of Mebeverine +Synbiotic combination therapy in patients with diarrhea predominant IBS. The study was carried for a period of one year in a Medical college in South India.
Table 1 depicts the general profile of the study patients. Out of the 132 patients screened, 60 patients who fulfilled the inclusion criteria were recruited for the study. They were randomized into three groups of 20 each and each group received respective medications for 12 weeks. It was observed that the mean age of the patients in Group A, B and C was 40.55 with SD 10.99, 39.3 with SD 12.33 and 38.05 with SD 10.2 respectively. However no statistical association was found between the age and the study groups $(\mathrm{p}=0.78)$.

Table 1: Depicts the age wise distribution of the study population.

\begin{tabular}{|llll|}
\hline Group & N & Mean age & SD \\
\hline A & 20 & 40.55 & 10.99 \\
\hline B & 20 & 39.3 & 12.338 \\
\hline C & 20 & 38.05 & 10.2 \\
\hline $\mathrm{P}=0.78$ & & & \\
\hline
\end{tabular}

Table 2 depicts the sex wise distribution of the study patients. It was found that majority of the study patients were males $(75 \%)$ in all the three groups as compared to the females $(25 \%)$ respectively. Further there was no statistical association between the gender of the patients and the study groups in which they were allocated $(\mathrm{p}=0.91)$.

Table 2: Depicts the sex wise distribution of the study population.

\begin{tabular}{|c|c|c|c|c|}
\hline \multirow{2}{*}{ Group } & \multicolumn{2}{|c|}{ Male } & \multicolumn{2}{|c|}{ Female } \\
\hline & $\mathbf{N}$ & $\%$ & $\mathbf{N}$ & $\%$ \\
\hline A & 15 & 75 & 5 & 25 \\
\hline B & 15 & 75 & 5 & 25 \\
\hline $\mathrm{C}$ & 14 & 70 & 6 & 30 \\
\hline $\mathrm{P}=0.91$ & & & & \\
\hline
\end{tabular}

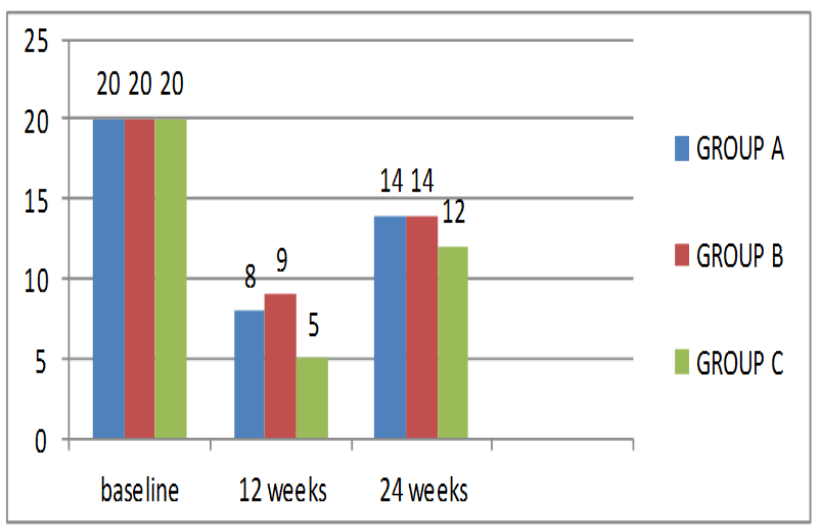

Figure 1: Frequency of abdominal pain among the study population.

Figure 1 reveals the frequency of abdominal pain among the study subjects. It was found that the percentage reduction in abdominal pain was higher in the 
combination group (75\%) when compared to Synbiotic $(55 \%)$ and Mebeverine (60\%) group.

Figure 2 reveals the mean stool frequency among the study subjects. Mean stool frequency showed better reduction in combination group (1.7) than Synbiotic (2.05) and Mebeverine (3.15) alone group. Comparison between the groups showed a statistically significant reduction in stool frequency in combination group $(\mathrm{p}=<0.001)$ and Synbiotic alone group $(\mathrm{p}=0.002)$ when compared with Mebeverine alone group.

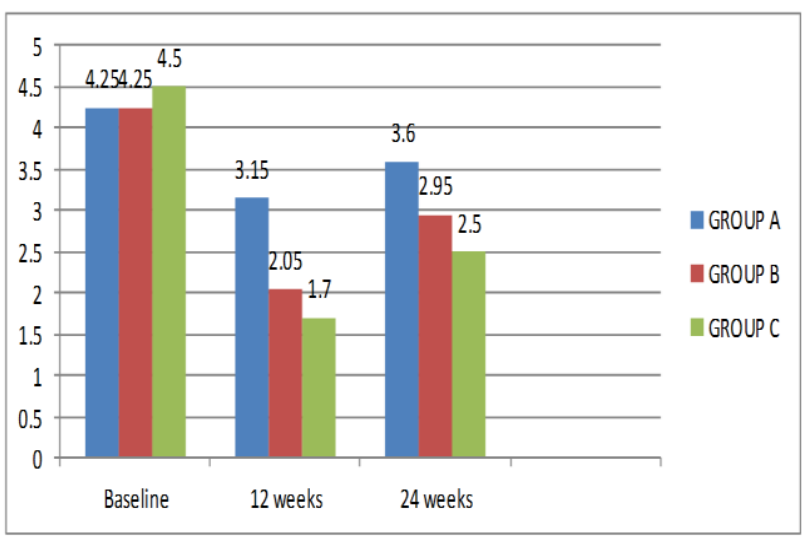

Figure 2: Mean stool frequency among the study population.

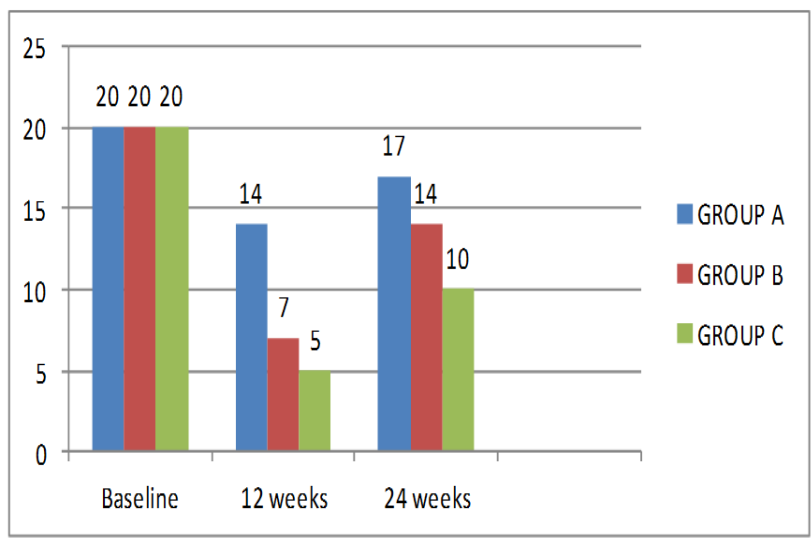

Figure 3: The stool consistency among the study population.

Figure 3 depicts the stool consistency among the study subjects. The percentage improvement in stool consistency was higher in combination group (75\%) when compared to Synbiotic alone $(65 \%)$ and Mebeverine alone (30\%). Comparison between the groups showed a statistically significant reduction in stool consistency in combination group $(\mathrm{p}=0.004)$ and Synbiotic alone group $(\mathrm{p}=0.027)$ when compared with Mebeverine alone group. This shows that combination group and Synbiotic alone group were better than Mebeverine alone group in reducing loose stools in IBS. On comparing between the groups, a statistically significant improvement in bloating symptoms was seen in combination group $(\mathrm{p}=0.038)$ when compared with Mebeverine alone group.

\section{DISCUSSION}

The present study was conducted to compare the efficacy of Mebeverine + Synbiotic combination therapy in patients with diarrhea predominant irritable bowel syndrome with Mebeverine and Synbiotic monotherapy. The efficacy was assessed based on symptom improvement during the treatment period and extension of duration of remission in the follow up period. Tolerability of the drug was analysed based on laboratory investigations and adverse event monitoring in the study period.

Studies conducted previously with Synbiotic alone showed that, Synbiotic significantly reduced stool frequency, abdominal pain and flatulence. In our study also there was similar improvement in stool frequency and abdominal pain and also similar to the study conducted earlier where Mebeverine was effective in reducing abdominal pain.

During the follow up period, the patients were followed for 12 weeks after completion of drug therapy to find out which treatment group had better remission of IBS symptoms. From the results of the follow up period it is evident that combination therapy had significant remission in stool frequency and stool consistency when compared with other groups.

At the end of the treatment period, the biochemical parameters did not show any statistically significant difference in the combination group when compared with the other groups. No adverse events were observed during the treatment period. There by suggesting that the drugs are safe when given in combination.

In the follow up period, combination of Synbiotic with Mebeverine showed better remission in stool frequency and stool consistency when compared to Mebeverine alone and Synbiotic alone group

\section{CONCLUSION}

Combination of Mebeverine + Synbiotic is more effective in improving most of the troublesome symptoms in patients with diarrhea predominant irritable bowel syndrome than Mebeverine and Synbiotic. This combination is effective in maintaining remission in terms of frequency and consistency of stools in patients with IBS. In addition, the combination of drugs did not have any adverse effects, thereby showing their safety.

Funding: No funding sources

Conflict of interest: None declared

Ethical approval: The study was approved by the Institutional Ethics Committee 


\section{REFERENCES}

1. Hahn BA, Yan S, Strassels S. Impact of irritable bowel syndrome on quality of life and resource use in the United States and United Kingdom. Digestion. 1999;60(1):77-81.

2. Malinen E, Rintilla T, Kajander K, Matto J, Kassinen A, Krogius L, et al. Analysis of the fecal microbiota of irritable bowel syndrome patients and healthy controls with real-time PCR. The American journal of gastroenterology. 2005;100(2):373-82.

3. King TS, Elia M, Hunter JO. Abnormal colonic fermentation in irritable Bowel syndrome. Lancet. 1998;352(9135):1187-9.

4. Treem WR, Ashan N, Kastoff G, Hyams JS. Fecal short chain fatty acids in patients with diarrhea predominant irritable bowel syndrome: in vitro studies of carbohydrate fermentation. Journal of pediatric gastroenterology and nutrition. 1996;23(3):280-6.

5. Chey WY, Jin HO, Lee MH. Colonic motility abnormality in patients with irritable bowel syndrome exhibiting abdominal pain and diarrhea. AmJ Gastroenterol. 2001;96:1499-506.
6. Clemens CH, Samson M, Roelofs JM. Association between pain episodes and high amplitude propagated pressure waves in patients with irritable bowel syndrome AmJ Gastroenterol. 2003;98:183843.

7. Kingham JGC, Bown R, Colson R, Clark ML. Jejunal motility in patients with functional abdominal pain. Gut. 1984;25:375.

8. Sharkey KA, Wallace JL. Treatment of bowel motility disorders. In: Laurence Brunton, Bruce Chabner, Bjorn Knollman (eds) Goodman and Gilman's, The pharmacological basis of therapeutics, $12^{\text {th }}$ ed:1339-1343.

9. Colin Drollery. Therapeutic Drugs $2^{\text {nd }}$ edition. Churchill Livingston. 1999;1:M15-7.

Cite this article as: Sujatha G, Dhanasekaran M, Qadri SS, Jeevithan S. A comparative study of mebeverine and synbiotic combination in patients with diarrhoea predominant Irritable Bowel Syndrome in a Medical College in South India. Int $\mathrm{J}$ Basic Clin Pharmacol 2017;6:12-6. 